PROCEEDINGS OF THE

AMERICAN MATHEMATICAL SOCIETY

Volume 140, Number 11, November 2012, Pages 3867-3881

S 0002-9939(2012)11221-3

Article electronically published on March 8, 2012

\title{
TWISTED COHOMOLOGY AND HOMOLOGY GROUPS ASSOCIATED TO THE RIEMANN-WIRTINGER INTEGRAL
}

\author{
TOSHIYUKI MANO AND HUMIHIKO WATANABE
}

(Communicated by Ted Chinburg)

Dedicated to Professor Keizo Yamaguchi on his sixtieth birthday

\begin{abstract}
We study twisted cohomology and homology groups on a onedimensional complex torus minus $n$ distinct points with coefficients in a certain local system of rank one. This local system comes from the integrand of the Riemann-Wirtinger integral introduced by Mano. We construct bases of nonvanishing cohomology and homology groups, give an interpretation as a pairing of a cohomology class and a homology class to the Riemann-Wirtinger integral, and finally describe briefly the Gauss-Manin connection on the cohomology groups.
\end{abstract}

\section{INTRODUCTION}

The twisted de Rham theory developed by Aomoto [1, 3], 6] has brought a unified treatment, and a systematic way of generalization, of various hypergeometric integrals which were invented and investigated by many authors. According to Aomoto, any such integral is interpreted as a pairing of a homology class and a cohomology class on a complex projective space $\mathbf{P}^{n}$ minus an effective divisor $D$ with coefficients in a local system of rank one, which is defined by a multi-valued function on $\mathbf{P}^{n}$ ramified just along $D$. Moreover, knowing the structures of the corresponding homology and cohomology groups enables us not only to produce systematically a system of differential equations satisfied by such integrals but also to determine the connection formulae and the monodromy representations of such integrals [2], 4], [5].

The purpose of this paper is to study twisted cohomology and homology groups on a one-dimensional complex torus minus $n$ distinct points. Let us formulate our problem in this paper. Let $\mathbf{C}$ be the additive group of complex numbers and $\Gamma$ the lattice in $\mathbf{C}$ generated by 1 and $\tau$ with $\operatorname{Im} \tau>0$. A one-dimensional complex torus is given by $E=E_{\tau}=\mathbf{C} / \Gamma$. Let $t_{1}, \ldots, t_{n}$ be $n$ distinct points of $E$. We set $M=M\left(t_{1}, \ldots, t_{n}, \tau\right)=E \backslash\left\{t_{1}, \ldots, t_{n}\right\}$. Let $T(u)$ be a multi-valued function on $M$ given by $T(u)=e^{2 \pi \sqrt{-1} c_{0} u} \theta\left(u-t_{1}\right)^{c_{1}} \cdots \theta\left(u-t_{n}\right)^{c_{n}}$, where $c_{i}(i=0, \ldots, n)$ denote

Received by the editors December 7, 2009 and, in revised form, August 21, 2010; January 31, 2011; April 19, 2011 and April 28, 2011.

2010 Mathematics Subject Classification. Primary 33C05; Secondary 14K25, 55N25, 14F40, $32 \mathrm{C} 35$.

Key words and phrases. Theta function, integral representation.

The first author was supported in part by GCOE, Kyoto University and MEXT Grant-in-Aid for Young Scientists (B) (No. 21740118).

The second author was supported in part by Grant-in-Aid for Scientific Research (C) (No. 19540158), JSPS.

(C)2012 American Mathematical Society 3867 
complex constants satisfying $c_{1}+\cdots+c_{n}=0$, and $\theta(u)$ denotes the theta function with $\theta(0)=0$ (see Notation for theta functions). Let $\mathcal{L}$ be the local system on $M$ defined by the multi-valuedness of $T(u)^{-1}$, and $\check{\mathcal{L}}$ be its dual: $\mathcal{L}=\mathbf{C} T(u)^{-1}$ and $\breve{\mathcal{L}}=\mathbf{C} T(u)$. For a complex number $\lambda$, let $R_{\lambda}$ be the local system of rank one on $E$ determined by the following one-dimensional representation $e_{\lambda}$ of the fundamental group of $E$ (which is isomorphic to $\Gamma$ ): $e_{\lambda}(1)=1, e_{\lambda}(\tau)=e^{2 \pi \sqrt{-1} \lambda}$. We denote by $\check{R}_{\lambda}$ its dual. By the standard argument (e.g. [11, $\S 9$ ), we can identify the local system $R_{\lambda}$ with a flat line bundle on $E$ (which we denote by the same letter $R_{\lambda}$ by abuse of notation) and assume that the parameter $\lambda$ runs over the Jacobian of $E$. Besides, we can regard a multi-valued function $\mathfrak{s}(u ; \lambda)$ defined in Notation for theta functions as a global meromorphic section of the line bundle $R_{\lambda}$. Then we can define the twisted cohomology and homology groups with coefficients in local systems: $H^{i}\left(M, \mathcal{L} \otimes_{\mathbf{C}} R_{\lambda}\right)$ and $H_{i}\left(M, \check{\mathcal{L}} \otimes_{\mathbf{C}} \check{R}_{\lambda}\right)$. The main purpose of this paper is to investigate their structures at length.

The study of these cohomology and homology groups gives us the foundation for analysis of integrals of the following type:

(1.1)

$f_{j}=\int_{\gamma} e^{2 \pi \sqrt{-1} c_{0} u} \theta\left(u-t_{1}\right)^{c_{1}} \cdots \theta\left(u-t_{n}\right)^{c_{n}} \mathfrak{s}\left(u-t_{j} ; \lambda\right) d u, j=1, \ldots, n, \gamma:$ a cycle,

which we call the Riemann-Wirtinger integral. According to Mano [14, [15, the integral (1.1) appears as a particular solution of a system of partial differential equations of the integrability condition of monodromy-preserving deformation of a Fuchsian differential equation with $n$ singularities $t_{1}, \ldots, t_{n}$ on a one-dimensional complex torus. Moreover, the integral (1.1) includes Gauss' hypergeometric function; namely, it is known [21] that, in the case where $n=4, \lambda=0, t_{1}=0, t_{2}=$ $1 / 2, t_{3}=\tau / 2, t_{4}=(1+\tau) / 2$, it reduces essentially to Euler's integral representation for Gauss' hypergeometric function. Therefore the integral (1.1) in this case is called in [17] the Wirtinger integral. In [19], 20] Watanabe studied the monodromy representation and the differential equations for the Wirtinger integral from the viewpoint of the theory of theta functions. As a natural and interesting generalization of the Wirtinger integral, we can also consider the integral (1.1) with the following conditions: $\lambda=0, n=N^{2}, N \geq 3$ and the set of singularities $\left\{t_{1}, \ldots, t_{n}\right\}$ coincides with that of $N$-torsion points on the torus (which we call the generalized Wirtinger integral of level $N$ ). For the generalized Wirtinger integrals, we are interested in solving the connection problem and the monodoromy representation and deriving differential equations satisfied by them. These topics will be discussed elsewhere. On the other hand the Riemann-Wirtinger integral is very similar to the integral representation constructed by Felder and Varchenko 9 which is an integral of a product of fractional powers of theta functions solving the elliptic Knizhnik-Zamolodchikov-Bernard (KZB) equation. We will compare these two integral representations in the present paper.

This paper is a natural generalization of the paper [18] for the Wirtinger integral, so several techniques used there are applied to the discussion in this paper. K. Ito [12] studied the twisted homology groups with coefficients in the local system defined by a multi-valued function of a special form on the complex torus minus four points. We can also regard this paper as a generalization of [12].

In Section 2, we study the twisted cohomology groups $H^{i}\left(M, \mathcal{L} \otimes_{\mathbf{C}} R_{\lambda}\right)$. The main task here is to determine the structure of the non-vanishing cohomology group 
$H^{1}\left(M, \mathcal{L} \otimes_{\mathbf{C}} R_{\lambda}\right)$. It changes according to whether $\lambda=0$ or $\lambda \in P \backslash\{0\}$, where $P=\{a \tau+b \in \mathbf{C} \mid 0 \leq a, b<1\}$ denotes a realization on the complex plane of the Jacobian of $E$ (Theorem 2.77). In the case where $\lambda \in P \backslash\{0\}$, similarly to the case of projective spaces (i.e., integrals of a product of fractional powers of rational functions), we can take a basis consisting of only logarithmic 1-forms. In the case where $\lambda=0$, however, we cannot find any basis consisting of only logarithmic 1forms, but we need a 1-form having a pole of order more than one to give its basis (Corollary 2.9). Proposition 2.5 is crucial to prove Theorem 2.7. Although this proposition has already been proved algebro-geometrically by Deligne [8, we give here another proof based on the theory of complex analysis (see Remark 2.6). As we have already encountered in the case of the Wirtinger integral [18, the phenomenon in Corollary 2.9 is new in the sense that the non-vanishing cohomology groups for complex projective spaces are described exclusively by logarithmic forms but the cohomology group for the complex torus is not necessarily described in such a way.

In Section 3, we study the twisted homology groups $H_{i}\left(M, \check{\mathcal{L}} \otimes_{\mathbf{C}} \check{R}_{\lambda}\right)$. By giving a cell decomposition for the space $M$, we choose twisted cycles as generators of this group and establish the linear relation satisfied by them explicitly (Theorem 3.1).

In Section 4, as an application of the results obtained in the preceding sections, we study the dependence of cohomology and homology groups with respect to the variables $t_{1}, \ldots, t_{n}, \tau$. Namely, we introduce a "base space" $\mathcal{T}$ parameterized by these data and take the union $\bigcup_{\left(t_{1}, \ldots, t_{n}, \tau\right) \in \mathcal{T}} H^{1}\left(M\left(t_{1}, \ldots, t_{n}, \tau\right), \mathcal{L} \otimes_{\mathbf{C}} R_{\lambda}\right)$. The tensor product of this union and the structure sheaf $\mathcal{O}_{\mathcal{T}}$ of $\mathcal{T}$ is, roughly speaking, equal to $R^{1} f_{*}\left(\mathcal{L} \otimes_{\mathbf{C}} R_{\lambda}\right) \otimes_{\mathbf{C}} \mathcal{O}_{\mathcal{T}}$, where $f$ denotes a natural projection of the total space $\mathcal{M}=\bigcup_{\left(t_{1}, \ldots, t_{n}, \tau\right) \in \mathcal{T}} M\left(t_{1}, \ldots, t_{n}, \tau\right)$ onto $\mathcal{T}$. To describe the GaussManin connection on $R^{1} f_{*}\left(\mathcal{L} \otimes_{\mathbf{C}} R_{\lambda}\right) \otimes_{\mathbf{C}} \mathcal{O}_{\mathcal{T}}$ ([13]), we establish the formulas of differentiation with respect to the variables $t_{1}, \ldots, t_{n}, \tau$ on $R^{1} f_{*}\left(\mathcal{L} \otimes_{\mathbf{C}} R_{\lambda}\right) \otimes_{\mathbf{C}} \mathcal{O}_{\mathcal{T}}$ (Proposition 4.1).

In Section 5, we refer to a relation between the Riemann-Wirtinger integral and Felder-Varchenko's integral solution of the KZB equation. Solutions of the KZB equation are generally represented by multiple integrals over direct products of elliptic curves. The Riemann-Wirtinger integral is related to the so-called one integral variable $\mathfrak{s l}_{2}$-case of the KZB equation. In Proposition 5.1, we give a system of partial differential equations which is a generalization of the KZB equation. Then we see that the Riemann-Wirtinger integral is another specialization of this system.

Notation for theta functions. In this paper, we follow Chandrasekharan's notation for theta functions [7]:

$$
\begin{aligned}
& \theta(u)=\theta(u, \tau)=-\sqrt{-1} \sum_{n=-\infty}^{+\infty}(-1)^{n} e^{\pi \sqrt{-1}(n+1 / 2)^{2} \tau} e^{2 \pi \sqrt{-1}(n+1 / 2) u}, \\
& \theta_{1}(u)=\theta_{1}(u, \tau)=\sum_{n=-\infty}^{+\infty} e^{\pi \sqrt{-1}(n+1 / 2)^{2} \tau} e^{2 \pi \sqrt{-1}(n+1 / 2) u}, \\
& \theta_{2}(u)=\theta_{2}(u, \tau)=\sum_{n=-\infty}^{+\infty}(-1)^{n} e^{\pi \sqrt{-1} n^{2} \tau} e^{2 \pi \sqrt{-1} n u}, \\
& \theta_{3}(u)=\theta_{3}(u, \tau)=\sum_{n=-\infty}^{+\infty} e^{\pi \sqrt{-1} n^{2} \tau} e^{2 \pi \sqrt{-1} n u} .
\end{aligned}
$$


Then $\theta(u)$ is an odd function and $\theta_{1}(u), \theta_{2}(u), \theta_{3}(u)$ are even functions. These functions are related to each other as follows:

$$
\begin{aligned}
& \theta_{1}(u)=\theta\left(u+\frac{1}{2}\right), \\
& \theta_{2}(u)=-\sqrt{-1} e^{\pi \sqrt{-1}(\tau / 4+u)} \theta\left(u+\frac{\tau}{2}\right), \\
& \theta_{3}(u)=e^{\pi \sqrt{-1}(\tau / 4+u)} \theta\left(u+\frac{\tau+1}{2}\right) .
\end{aligned}
$$

We also use the following symbols: $\theta^{\prime}=\theta^{\prime}(0, \tau), \theta_{i}=\theta_{i}(0, \tau), i=1,2,3$. We introduce the following function:

$$
\mathfrak{s}(u ; \lambda)=\frac{\theta(u-\lambda) \theta^{\prime}}{\theta(u) \theta(-\lambda)} .
$$

Then $\mathfrak{s}(u ; \lambda)$ has the quasi-periodicity

$$
\mathfrak{s}(u+1 ; \lambda)=\mathfrak{s}(u ; \lambda), \quad \mathfrak{s}(u+\tau ; \lambda)=e^{2 \pi \sqrt{-1} \lambda} \mathfrak{s}(u ; \lambda) .
$$

Moreover we use the symbol $\rho(u)=\theta^{\prime}(u) / \theta(u)$.

\section{TWisted COHOMOLOGY GROUPS}

Let $\mathbf{H}$ be the upper half plane. For $\tau \in \mathbf{H}$, we set $\Gamma=\Gamma_{\tau}=\mathbf{Z}+\mathbf{Z} \tau$, a subgroup of the additive group $\mathbf{C}$ of complex numbers, where $\mathbf{Z}$ is the additive group of integers. The fundamental group of the torus $E=E_{\tau}=\mathbf{C} / \Gamma$ is isomorphic to the group $\Gamma$. Let $\lambda$ be a complex number. We define a one-dimensional representation of the fundamental group, which we denote by $e_{\lambda}: \Gamma \ni \gamma \rightarrow e_{\lambda}(\gamma) \in \mathbf{C}^{*}\left(\mathbf{C}^{*}\right.$ denotes the multiplicative group of non-zero complex numbers), by the assignment of generators of $\Gamma$ into $\mathbf{C}^{*}: e_{\lambda}(1)=1, e_{\lambda}(\tau)=e^{2 \pi \sqrt{-1} \lambda}$. Let $R_{\lambda}$ be the local system of rank one on $E$ determined by this representation $e_{\lambda}$. Let $\mathcal{O}_{E}$ be the sheaf of holomorphic functions on $E$. We set $\mathcal{O}_{E, \lambda}=\mathcal{O}_{E} \otimes_{\mathbf{C}} R_{\lambda}$, the tensor product of $\mathcal{O}_{E}$ and $R_{\lambda}$. Let $L_{\lambda}$ be the line bundle on $E$ whose local sections are generated by the sheaf $\mathcal{O}_{E, \lambda}$. The sheaf $\mathcal{O}_{E, \lambda}$ is also denoted by $\mathcal{O}_{E}\left(L_{\lambda}\right)$ in the literature. Then we have

Lemma 2.1. Assume that $\lambda \notin \Gamma$. Then $H^{0}\left(E, \mathcal{O}_{E, \lambda}\right)=H^{1}\left(E, \mathcal{O}_{E, \lambda}\right)=0$.

Proof. Let $f$ be in $H^{0}\left(E, \mathcal{O}_{E, \lambda}\right)$. By definition, $f(u)$ is holomorphic on $E$, satisfying the relations $f(u+1)=f(u)$ and $f(u+\tau)=e^{2 \pi \sqrt{-1} \lambda} f(u)$. By the definition of $\mathfrak{s}(u ;-\lambda)$, the product $f(u) \mathfrak{s}(u ;-\lambda)$ must be an elliptic function on $E$ with at most a single simple pole at $u=0$, from which we have $f=0$. Therefore $H^{0}\left(E, \mathcal{O}_{E, \lambda}\right)=0$. Now the Riemann-Roch theorem holds: $\operatorname{dim} H^{0}\left(E, \mathcal{O}_{E, \lambda}\right)-\operatorname{dim} H^{1}\left(E, \mathcal{O}_{E, \lambda}\right)-$ $c\left(L_{\lambda}\right)=1-g$, where $c\left(L_{\lambda}\right)$ is the first Chern class of the line bundle $L_{\lambda}$. Since we can take $\mathfrak{s}(u ; \lambda)$ as a global meromorphic section of $L_{\lambda}$, we conclude that $c\left(L_{\lambda}\right)=0$. Since $g=1$ and $H^{0}\left(E, \mathcal{O}_{E, \lambda}\right)=0$, we have $\operatorname{dim} H^{1}\left(E, \mathcal{O}_{E, \lambda}\right)=0$ by the RiemannRoch theorem, which completes the proof of Lemma 2.1

Let $t_{1}, \ldots, t_{n}$ be $n$ distinct points of $E$, where $n \geq 2$. We set $D=\left\{t_{1}, \ldots, t_{n}\right\}$ and $M=M\left(t_{1}, \ldots, t_{n}, \tau\right)=E \backslash D$. Let $\mathcal{U}=\left\{U_{i}\right\}_{i \in \Lambda}$ be an open covering of $E$. Without loss of generality we may assume that $\mathcal{U}$ is a Leray open covering such that every open set $U_{i} \in \mathcal{U}$ contains at most one point $t_{k}$ of $D$. Let us consider a collection $\mu=\left\{h_{i}\right\}_{i \in \Lambda}$, where $h_{i}$ is a holomorphic section of $L_{\lambda}$ on $U_{i}$ if $U_{i}$ contains no point of $D$, or it is a holomorphic section of $L_{\lambda}$ on $U_{i}-\left\{t_{k}\right\}$ and 
may have an isolated singularity (i.e., a pole or an essential singularity) at $t_{k}$ if $U_{i}$ contains a point $t_{k} \in D$. Such a collection $\mu$ is called a Mittag-Leffler distribution if, $\rho_{U, V}$ denoting the restriction map of $H^{0}\left(U, \mathcal{O}_{E, \lambda}\right)$ to $H^{0}\left(V, \mathcal{O}_{E, \lambda}\right)$ for $U \supset V$, the difference $\rho_{U_{i}, U_{i} \cap U_{j}}\left(h_{i}\right)-\rho_{U_{j}, U_{i} \cap U_{j}}\left(h_{j}\right)$ is holomorphic on $U_{i} \cap U_{j}$. By a solution of $\mu$ we mean a global analytic section $h$ of $L_{\lambda}$ holomorphic on $M$ such that the difference $\rho_{E, U_{i}}(h)-h_{i}$ is a holomorphic section on $U_{i}$ for every $i \in \Lambda$. Here we note that our definition of a Mittag-Leffler distribution is a little wider than the one given in [10]. Then we have

Lemma 2.2. If $\lambda \notin \Gamma$, every Mittag-Leffler distribution $\mu$ has a solution.

Proof. We set $\left.h_{i}\right|_{U_{i} \cap U_{j}}=\rho_{U_{i}, U_{i} \cap U_{j}}\left(h_{i}\right)$. The collection $\left\{\left.h_{i}\right|_{U_{i} \cap U_{j}}-\left.h_{j}\right|_{U_{i} \cap U_{j}}\right\}_{i \neq j}$ forms a 1-cocycle: $\left\{\left.h_{i}\right|_{U_{i} \cap U_{j}}-\left.h_{j}\right|_{U_{i} \cap U_{j}}\right\}_{i \neq j} \in Z^{1}\left(\mathcal{U}, \mathcal{O}_{E, \lambda}\right)$. Since $H^{1}\left(E, \mathcal{O}_{E, \lambda}\right)=0$ by Lemma 2.1 and $\mathcal{U}$ is a Leray covering, there exists a local holomorphic section $g_{i}$ on every $U_{i}$ such that $\left.h_{i}\right|_{U_{i} \cap U_{j}}-\left.h_{j}\right|_{U_{i} \cap U_{j}}=\left.g_{i}\right|_{U_{i} \cap U_{j}}-\left.g_{j}\right|_{U_{i} \cap U_{j}}$. Then we have $\left.h_{i}\right|_{U_{i} \cap U_{j}}-\left.g_{i}\right|_{U_{i} \cap U_{j}}=\left.h_{j}\right|_{U_{i} \cap U_{j}}-\left.g_{j}\right|_{U_{i} \cap U_{j}}$. Gluing the local sections $h_{i}-g_{i}$ over $E$, we have a global section on $E$, which is the desired solution of $\mu$.

When $\lambda \in \Gamma$, we may assume $\lambda=0$ without loss of generality.

Lemma 2.3. Assume that $\lambda=0$. Let $a_{k}(1 \leq k \leq n)$ be the residue of the 1-form $h_{i} d u$ at the singularity $u=t_{k}$ if $t_{k} \in U_{i}$. Then the Mittag-Leffler distribution $\mu$ has a solution if and only if $\sum_{k=1}^{n} a_{k}=0$.

For the proof, see [10], Chapter 2, $§ 18$.

Let $\mathcal{O}_{M}$ be the sheaf of holomorphic functions on $M$ and let $\Omega_{M}^{1}$ be the sheaf of holomorphic 1-forms on $M$. We define the sheaves $\mathcal{O}_{\lambda}$ and $\Omega_{\lambda}^{1}$ on $M$ by $\mathcal{O}_{\lambda}=$ $\mathcal{O}_{M} \otimes_{\mathbf{C}} R_{\lambda}$ and $\Omega_{\lambda}^{1}=\Omega_{M}^{1} \otimes_{\mathbf{C}} R_{\lambda}$, where we denote the restriction of $R_{\lambda}$ to $M$ by the same symbol $R_{\lambda}$ by abuse of notation. Since $R_{\lambda}$ is locally constant and without torsion, we have the exact sequence of sheaves on $M$ :

$$
0 \rightarrow R_{\lambda} \rightarrow \mathcal{O}_{\lambda} \stackrel{d}{\longrightarrow} \Omega_{\lambda}^{1} \rightarrow 0
$$

where $d$ denotes the sheaf mapping induced by the differential $d: \mathcal{O}_{M} \rightarrow \Omega_{M}^{1}$. Let $c_{0}$ be an arbitrary complex number, and $c_{1}, \ldots, c_{n}(n \geq 2)$ be non-integral complex numbers satisfying $c_{1}+\cdots+c_{n}=0$. We define a multi-valued function $T(u)$ on $M$ by $T(u)=e^{2 \pi \sqrt{-1} c_{0} u} \theta\left(u-t_{1}\right)^{c_{1}} \cdots \theta\left(u-t_{n}\right)^{c_{n}}$. We set $\omega=d(\log T(u))$. We define a connection $\nabla$ by $\nabla \varphi=d \varphi+\omega \wedge \varphi$. Then we have $\nabla \nabla=0$ and $\nabla(1)=\omega$. The connection $\nabla$ defines a sheaf morphism $\mathcal{O}_{M} \rightarrow \Omega_{M}^{1}$, and therefore $\mathcal{O}_{\lambda} \rightarrow \Omega_{\lambda}^{1}$. Let $\mathcal{L}$ and $\check{\mathcal{L}}$ be the local systems on $M$ defined by the multi-valuedness of $T(u)^{-1}$ and $T(u)$, respectively: $\mathcal{L}=\mathbf{C} T(u)^{-1}$ and $\check{\mathcal{L}}=\mathbf{C} T(u)$. They are dual to each other. In the case where we emphasize the dependence on the parameters $c_{i}$ contained in $\mathcal{L}$ and $\check{\mathcal{L}}$, we also write $\mathcal{L}=\mathcal{L}\left(c_{0}, \ldots, c_{n}\right)$ and $\check{\mathcal{L}}=\check{\mathcal{L}}\left(c_{0}, \ldots, c_{n}\right)$. Since the local system $\mathcal{L}$ is locally constant and without torsion, we have the following exact sequence on $M$ :

$$
0 \rightarrow \mathcal{L} \otimes_{\mathbf{C}} R_{\lambda} \rightarrow \mathcal{L} \otimes_{\mathbf{C}} \mathcal{O}_{\lambda} \stackrel{\text { id } \otimes_{d}}{\longrightarrow} \mathcal{L} \otimes_{\mathbf{C}} \Omega_{\lambda}^{1} \rightarrow 0 .
$$

Let $\varphi$ be a local section of $\mathcal{O}_{\lambda}$. Then the assignment $\varphi \mapsto T(u) \varphi$ defines a sheaf isomorphism $\mathcal{O}_{\lambda} \rightarrow \mathcal{L} \otimes_{\mathbf{C}} \mathcal{O}_{\lambda}$. Since $d(T(u) \varphi)=T(u) \nabla \varphi$, the following diagram is 
commutative:

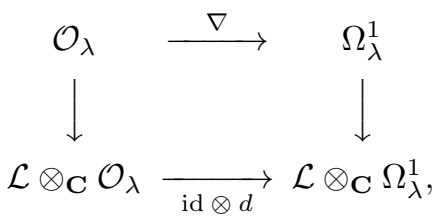

where the vertical arrows represent isomorphisms. Combining this commutative diagram and the exact sequence (2.1), we have the exact sequence

$$
0 \rightarrow \mathcal{L} \otimes_{\mathbf{C}} R_{\lambda} \rightarrow \mathcal{O}_{\lambda} \stackrel{\nabla}{\longrightarrow} \Omega_{\lambda}^{1} \rightarrow 0,
$$

from which we have the following long exact sequence of the cohomology groups:

$$
\begin{gathered}
0 \rightarrow H^{0}\left(M, \mathcal{L} \otimes_{\mathbf{C}} R_{\lambda}\right) \rightarrow H^{0}\left(M, \mathcal{O}_{\lambda}\right) \stackrel{\nabla}{\longrightarrow} H^{0}\left(M, \Omega_{\lambda}^{1}\right) \rightarrow H^{1}\left(M, \mathcal{L} \otimes_{\mathbf{C}} R_{\lambda}\right) \\
\rightarrow H^{1}\left(M, \mathcal{O}_{\lambda}\right) \stackrel{\nabla}{\longrightarrow} H^{1}\left(M, \Omega_{\lambda}^{1}\right) \rightarrow H^{2}\left(M, \mathcal{L} \otimes_{\mathbf{C}} R_{\lambda}\right) \rightarrow H^{2}\left(M, \mathcal{O}_{\lambda}\right) \\
\stackrel{\nabla}{\longrightarrow} H^{2}\left(M, \Omega_{\lambda}^{1}\right) \rightarrow \cdots .
\end{gathered}
$$

Proposition 2.4. We have $H^{i}\left(M, \mathcal{L} \otimes_{\mathbf{C}} R_{\lambda}\right)=0$ for $i \neq 1$, and

$$
H^{1}\left(M, \mathcal{L} \otimes_{\mathbf{C}} R_{\lambda}\right) \cong H^{0}\left(M, \Omega_{\lambda}^{1}\right) / \nabla\left(H^{0}\left(M, \mathcal{O}_{\lambda}\right)\right) .
$$

Proof. The local system $\mathcal{L} \otimes_{\mathbf{C}} R_{\lambda}$ has no global section on $M$ but the zero section. So we have $H^{0}\left(M, \mathcal{L} \otimes_{\mathbf{C}} R_{\lambda}\right)=0$. Note that $\mathcal{O}_{\lambda}$ and $\Omega_{\lambda}^{1}$ are coherent $\mathcal{O}_{M}$-modules. Since the open Riemann surface $M$ is Stein, we have $H^{i}\left(M, \mathcal{O}_{\lambda}\right)=H^{i}\left(M, \Omega_{\lambda}^{1}\right)=$ $0(i>0)$. Combining these results with (2.2), we have the short exact sequence

$$
0 \rightarrow H^{0}\left(M, \mathcal{O}_{\lambda}\right) \stackrel{\nabla}{\longrightarrow} H^{0}\left(M, \Omega_{\lambda}^{1}\right) \rightarrow H^{1}\left(M, \mathcal{L} \otimes_{\mathbf{C}} R_{\lambda}\right) \rightarrow 0
$$

and $H^{i}\left(M, \mathcal{L} \otimes_{\mathbf{C}} R_{\lambda}\right)=0(i \geq 2)$, from which the proposition follows.

Let $\tilde{\mathcal{K}}^{0}$ be the sheaf of meromorphic functions on $E$, and $\tilde{\mathcal{K}}^{1}$ be the sheaf of meromorphic 1-forms on $E$. Let $\mathcal{K}^{0}=H^{0}\left(E, \tilde{\mathcal{K}}^{0}\right)$ be the field of global meromorphic functions on $E$, and $\mathcal{K}^{1}=H^{0}\left(E, \tilde{\mathcal{K}}^{1}\right)$ be the $\mathcal{K}^{0}$-vector space of global meromorphic 1-forms on $E$. We define sheaves $\tilde{\mathcal{K}}_{\lambda}^{p}(p=0,1)$ on $E$ by $\tilde{\mathcal{K}}_{\lambda}^{p}=\tilde{\mathcal{K}}^{p} \otimes_{\mathbf{C}} R_{\lambda}$. Let $\mathcal{S}^{p}$ $(p=0,1)$ be the sheaf on $M$ whose sections on an open set $U \subset M$ form the C-vector space $\mathcal{S}^{p}(U)=\left\{s \in \mathcal{K}^{p} \mid s\right.$ is holomorphic on $\left.U\right\}$. By definition, $\mathcal{S}^{0}$ is a subsheaf of $\mathcal{O}_{M}$, and $\mathcal{S}^{1}$ a subsheaf of $\Omega_{M}^{1}$. We define sheaves $\mathcal{S}_{\lambda}^{0}$ and $\mathcal{S}_{\lambda}^{1}$ on $M$ by $\mathcal{S}_{\lambda}^{0}=\mathcal{S}^{0} \otimes_{\mathbf{C}} R_{\lambda}$ and $\mathcal{S}_{\lambda}^{1}=\mathcal{S}^{1} \otimes_{\mathbf{C}} R_{\lambda}$. Let us consider the subcomplex of sheaves

$$
0 \rightarrow \mathcal{S}_{\lambda}^{0} \stackrel{\nabla}{\longrightarrow} \mathcal{S}_{\lambda}^{1} \rightarrow 0
$$

of the complex

$$
0 \rightarrow \mathcal{O}_{\lambda} \stackrel{\nabla}{\longrightarrow} \Omega_{\lambda}^{1} \rightarrow 0 .
$$

The inclusion $\iota$ of (2.4) to (2.5) induces the natural homomorphism $\iota_{*}$ of the de Rham cohomologies:

$$
\iota_{*}: H^{0}\left(M, \mathcal{S}_{\lambda}^{1}\right) / \nabla\left(H^{0}\left(M, \mathcal{S}_{\lambda}^{0}\right)\right) \rightarrow H^{0}\left(M, \Omega_{\lambda}^{1}\right) / \nabla\left(H^{0}\left(M, \mathcal{O}_{\lambda}\right)\right) .
$$

Let $\mathcal{O}_{E}(* D)$ be the sheaf of functions meromorphic on $E$ and holomorphic on $M$, and $\Omega_{E}^{1}(* D)$ be the sheaf of 1-forms meromorphic on $E$ and holomorphic on $M$. We set $\mathcal{O}_{\lambda}(* D)=\mathcal{O}_{E}(* D) \otimes_{\mathbf{C}} R_{\lambda}$ and $\Omega_{\lambda}^{1}(* D)=\Omega_{E}^{1}(* D) \otimes_{\mathbf{C}} R_{\lambda}$. Since 
$H^{0}\left(M, \mathcal{S}_{\lambda}^{1}\right)=H^{0}\left(E, \Omega_{\lambda}^{1}(* D)\right)$ and $H^{0}\left(M, \mathcal{S}_{\lambda}^{0}\right)=H^{0}\left(E, \mathcal{O}_{\lambda}(* D)\right)$, 2.6. is written by

$$
\iota_{*}: H^{0}\left(E, \Omega_{\lambda}^{1}(* D)\right) / \nabla\left(H^{0}\left(E, \mathcal{O}_{\lambda}(* D)\right)\right) \rightarrow H^{0}\left(M, \Omega_{\lambda}^{1}\right) / \nabla\left(H^{0}\left(M, \mathcal{O}_{\lambda}\right)\right)
$$

In fact we have

Proposition 2.5. For any $\lambda \in \mathbf{C}, \iota_{*}$ is an isomorphism.

Proof. Since we have

$$
\mathcal{L}\left(c_{0}, c_{1}, \ldots, c_{n}\right) \otimes_{\mathbf{C}} R_{\lambda+m \tau+n} \cong \mathcal{L}\left(c_{0}+m, c_{1}, \ldots, c_{n}\right) \otimes_{\mathbf{C}} R_{\lambda},
$$

for $m, n \in \mathbf{Z}$, we may assume that $\lambda \in P=\{a \tau+b \mid 0 \leq a, b<1\}$ without loss of generality. For the proof we need the following claim:

(*) For $\varphi \in H^{0}\left(M, \Omega_{\lambda}^{1}\right)$, there exist $\psi \in H^{0}\left(E, \Omega_{\lambda}^{1}(* D)\right)$ and $\tilde{Q}_{*} \in H^{0}\left(M, \mathcal{O}_{\lambda}\right)$ such that $\varphi=\psi+\nabla \tilde{Q}_{*}$.

In fact, let $\varphi$ be in $H^{0}\left(M, \Omega_{\lambda}^{1}\right)$. Since the canonical line bundle of $E$ is trivial, we may set $\varphi=f(u) d u$, where $f(u)$ is a section belonging to $H^{0}\left(M, \mathcal{O}_{\lambda}\right)$ and may have isolated essential singularities at $u=t_{k}(k=1, \ldots, n)$. Let $P_{k}(u)$ be the principal part of the Laurent expansion of $f(u)$ at $u=t_{k}$. Let us find a function $Q_{k}(u)$ single-valued around $u=t_{k}$ satisfying the equation $P_{k}(u) d u=\nabla Q_{k}$, that is,

$$
P_{k}(u)=\frac{d Q_{k}}{d u}+Q_{k} \frac{d}{d u}(\log T(u))
$$

Here we may assume that $P_{k}(u)=\sum_{n \leq-1} a_{n}^{(k)}\left(u-t_{k}\right)^{n}$. By quadrature we have a general solution of this equation: $Q_{k}=T(u)^{-1}\left[\int T(u) P_{k}(u) d u+C\right]$ for some constant $C$. Since $Q_{k}$ is single-valued at $t_{k}$, the condition $C=0$ is necessary. Let us investigate the behaviour of the solution $Q_{k}=Q_{k}(u)$ with $C=0$ around $u=t_{k}$. Since $c_{k}$ is not an integer, the multi-valuedness of $T(u)$ around $u=$ $t_{k}$ comes from the factor $\left(u-t_{k}\right)^{c_{k}}$. Then we can write $T(u)=\left(u-t_{k}\right)^{c_{k}} \times$ (single-valued holomorphic function) around $u=t_{k}$. Moreover, since we can write $T(u) P_{k}(u)=\sum_{n=-\infty}^{+\infty} e_{n}^{(k)}\left(u-t_{k}\right)^{c_{k}+n}$ around $u=t_{k}$, we have

$$
\int T(u) P_{k}(u) d u=\sum_{n=-\infty}^{+\infty} \frac{e_{n}^{(k)}}{c_{k}+n+1}\left(u-t_{k}\right)^{c_{k}+n+1},
$$

which is of the form $\left(u-t_{k}\right)^{c_{k}} \times($ single-valued analytic function which may have an isolated singularity at $\left.u=t_{k}\right)$ around $u=t_{k}$. Consequently, the function $Q_{k}(u)=$ $T(u)^{-1} \int T(u) P_{k}(u) d u$ is a single-valued analytic function around $u=t_{k}$ which may have an isolated singularity at $u=t_{k}$ and therefore can be expanded in a Laurent series at $u=t_{k}$. We set $Q_{k}(u)=\sum_{n=-\infty}^{+\infty} b_{n}^{(k)}\left(u-t_{k}\right)^{n}$, the Laurent expansion at $u=t_{k}$. Moreover we set $Q_{k-}(u)=\sum_{n \leq 0} b_{n}^{(k)}\left(u-t_{k}\right)^{n}$ and $Q_{k+}(u)=$ $\sum_{n>1} b_{n}^{(k)}\left(u-t_{k}\right)^{n}$. Substituting $Q_{k}=Q_{k-}+Q_{k+}$ into the original equation above, we have $P_{k}=Q_{k-}^{\prime}+Q_{k+}^{\prime}+Q_{k-} \cdot(\log T(u))^{\prime}+Q_{k+} \cdot(\log T(u))^{\prime}$. Since $(\log T(u))^{\prime}$ has a pole of order one at $u=t_{k}$ and $Q_{k+}$ has a zero of order one at $u=t_{k}$, the product $Q_{k+} \cdot(\log T(u))^{\prime}$ is holomorphic at $u=t_{k}$, and so is $Q_{k+}^{\prime}$. Consequently, we see that in the right-hand side of the preceding relation the sum $Q_{k_{-}}^{\prime}+Q_{k_{-}} \cdot(\log T(u))^{\prime}$ contributes to the principal part $P_{k}$. Therefore, setting $\nabla Q_{k-}=g_{k}(u) d u$, we see that the principal part of the Laurent expansion of $g_{k}(u)$ at $u=t_{k}$ is equal to $P_{k}$. Let $\mathcal{U}=\left\{U_{i}\right\}_{i \in \Lambda}$ be a Leray open covering of $E$ such that every open set $U_{i}$ 
contains at most one point $t_{k}$ of $D$. To conclude the proof of the claim $(*)$, we consider the two cases: (i) $\lambda \neq 0$ and (ii) $\lambda=0$.

(i) Assume $\lambda \neq 0$. Let $\mu=\left\{Q_{* i}\right\}_{i \in \Lambda}$ be a Mittag-Leffler distribution subordinate to the open covering $\mathcal{U}$ consisting of local sections $Q_{* i}$ of $L_{\lambda}$ on $U_{i}$ such that for every open set $U_{i}$ containing $t_{k} \in D$ the section $Q_{* i}$ coincides with the branch containing $Q_{k-}-b_{0}^{(k)}$ and for every open set $U_{j}$ not containing any point of $D$ the section $Q_{* j}$ is holomorphic. Then, by Lemma 2.2, there exists a global analytic section $Q_{*}$ of $L_{\lambda}$ on $E$, holomorphic on $M$ such that the difference $Q_{k-}-b_{0}^{(k)}-Q_{*}$ is holomorphic around $u=t_{k}$. We note that for every $k$ the 1 -form $P_{k}(u) d u-\nabla b_{0}^{(k)}-\nabla Q_{*}$ is holomorphic at $u=t_{k}$ and that the 1-form $\nabla b_{0}^{(k)}$ locally defined around $u=t_{k}$ has a pole of order one at $u=t_{k}$ with residue $c_{k} b_{0}^{(k)}$. We set $\psi=\sum_{k=1}^{n} b_{0}^{(k)} c_{k} \mathfrak{s}\left(u-t_{k}, \lambda\right) d u \in$ $H^{0}\left(E, \Omega_{\lambda}^{1}(* D)\right)$. Then the 1-form $f(u) d u-\psi-\nabla Q_{*}$ is a global holomorphic section on $E$, and therefore by Lemma 2.1 we have $f(u) d u=\psi+\nabla Q_{*}$, which proves the claim $(*)$.

(ii) Assume $\lambda=0$. Then the claim $(*)$ follows if we apply Lemma 2.3 and the reasoning of the proof of Lemma 2.2 in $[18$. We omit the details.

We are now in a position to prove the proposition with the aid of the claim $(*)$. Let us take $[\varphi] \in H^{0}\left(M, \Omega_{\lambda}^{1}\right) / \nabla\left(H^{0}\left(M, \mathcal{O}_{\lambda}\right)\right)$ arbitrarily, where $\varphi \in H^{0}\left(M, \Omega_{\lambda}^{1}\right)$. If we form $[\psi] \in H^{0}\left(E, \Omega_{\lambda}^{1}(* D)\right) / \nabla\left(H^{0}\left(E, \mathcal{O}_{\lambda}(* D)\right)\right)$ from the element $\psi \in H^{0}(E$, $\left.\Omega_{\lambda}^{1}(* D)\right)$ whose existence is guaranteed by the claim $(*)$, then we have $\iota_{*}[\psi]=[\varphi]$, which proves the surjectivity of $\iota_{*}$. The proof of the injectivity of $\iota_{*}$ is as follows. For $[\psi] \in H^{0}\left(E, \Omega_{\lambda}^{1}(* D)\right) / \nabla\left(H^{0}\left(E, \mathcal{O}_{\lambda}(* D)\right)\right)$, assume that $\iota_{*}[\psi]=0$. We set $\psi=f(u) d u$, where $f(u)$ is a section of $H^{0}\left(E, \mathcal{O}_{\lambda}(* D)\right)$ that may have poles at points of $D$ if $f(u)$ is not holomorphic there. The equation $\iota_{*}[\psi]=0$ is translated into the assertion that there exists a section $g \in H^{0}\left(M, \mathcal{O}_{\lambda}\right)$ such that $f(u) d u=\nabla g$. The equation is rewritten as

$$
f(u)=\frac{d g}{d u}+g(u) \frac{d}{d u}(\log T(u))
$$

from which we have the solution $g(u)=T(u)^{-1} \int T(u) f(u) d u$. By the same argument as when we constructed $Q_{k}$ from $P_{k}$ and investigated the behaviour of $Q_{k}$ at $u=t_{k}$, we see that $g(u)$ is in $H^{0}\left(E, \mathcal{O}_{\lambda}(* D)\right)$, and $[\psi]=0$ as the equality in $H^{0}\left(E, \Omega_{\lambda}^{1}(* D)\right) / \nabla\left(H^{0}\left(E, \mathcal{O}_{\lambda}(* D)\right)\right)$, which proves the injectivity of $\iota_{*}$.

Remark 2.6. It is well known that this proposition is proved algebro-geometrically by Deligne's comparison theorem [8], II, Section 6 . On the other hand, to study the structure of the quotient group $H^{0}\left(E, \Omega_{\lambda}^{1}(* D)\right) / \nabla H^{0}\left(E, \mathcal{O}_{\lambda}(* D)\right)$ at length, we have to choose an effective divisor $D$ such that the vector space $H^{0}\left(E, \Omega_{\lambda}^{1}(D)\right)$ maps surjectively to the above group. In the analogous theory of integrals on complex projective spaces [6] one can choose for such a $D$ the "reduced" one, that is, the effective divisor all of whose integral coefficients are equal to one. In our case, however, it is indispensable to choose a non-reduced divisor $D^{\prime}=2\left[t_{1}\right]+\left[t_{2}\right]+\cdots+$ $\left[t_{n}\right]$ especially when $\lambda=0$ (cf. Theorem 2.7). Our proof of this proposition tells us what divisor $D$ is suitable to take for establishing the surjection of $H^{0}\left(E, \Omega_{\lambda}^{1}(D)\right)$ onto $H^{0}\left(E, \Omega_{\lambda}^{1}(* D)\right) / \nabla H^{0}\left(E, \mathcal{O}_{\lambda}(* D)\right)$. 
Let $D$ and $D^{\prime}$ be the divisors on $E$ defined by $D=\left[t_{1}\right]+\cdots+\left[t_{n}\right]$ and $D^{\prime}=$ $2\left[t_{1}\right]+\left[t_{2}\right]+\cdots+\left[t_{n}\right]$. Then we have the following natural homomorphisms:

$$
\begin{gathered}
I: H^{0}\left(E, \Omega_{\lambda}^{1}(D)\right) \rightarrow H^{0}\left(E, \Omega_{\lambda}^{1}(* D)\right) / \nabla H^{0}\left(E, \mathcal{O}_{\lambda}(* D)\right), \\
I^{\prime}: H^{0}\left(E, \Omega_{\lambda}^{1}\left(D^{\prime}\right)\right) \rightarrow H^{0}\left(E, \Omega_{\lambda}^{1}(* D)\right) / \nabla H^{0}\left(E, \mathcal{O}_{\lambda}(* D)\right),
\end{gathered}
$$

where, for a divisor $F$ on $E$, we denote $H^{0}\left(E, \Omega_{\lambda}^{p}(F)\right)=\left\{f \in H^{0}\left(E, \tilde{\mathcal{K}}_{\lambda}^{p}\right) \mid(f)+F \geq\right.$ $0\}$.

Theorem 2.7. Under the assumptions $c_{i} \notin \mathbf{Z}, i=1, \ldots, n$, the following assertions hold:

(i) For $\lambda \in P \backslash\{0\}$, I is an isomorphism.

(ii) For $\lambda \in P, I^{\prime}$ is surjective and $\operatorname{dim} \operatorname{ker} I^{\prime}=1$.

Proof. First, we prove (i). We introduce a filtration to the vector spaces $H^{0}(E$, $\left.\Omega_{\lambda}^{p}(* D)\right)(p=0,1)$ by the following:

$$
F_{k}=F_{k} H^{0}\left(E, \Omega_{\lambda}^{p}(* D)\right)=H^{0}\left(E, \Omega_{\lambda}^{p}(k D)\right), \quad k=0,1,2, \ldots
$$

It is obvious that

$$
\begin{aligned}
& F_{k} H^{0}\left(E, \Omega_{\lambda}^{p}(* D)\right) \subset F_{k+1} H^{0}\left(E, \Omega_{\lambda}^{p}(* D)\right), \\
& \bigcup_{k=0}^{\infty} F_{k} H^{0}\left(E, \Omega_{\lambda}^{p}(* D)\right)=H^{0}\left(E, \Omega_{\lambda}^{p}(* D)\right),
\end{aligned}
$$

and

$$
F_{0} H^{0}\left(E, \Omega_{\lambda}^{p}(* D)\right)=H^{0}\left(E, \Omega_{\lambda}^{p}\right)=0 .
$$

For $k \geq 1$, it is easy to see that the set of $n$ functions $\left\{\frac{\partial^{k-1}}{\partial u^{k-1}} \mathfrak{s}\left(u-t_{i} ; \lambda\right)\right\}_{i=1}^{n}$ forms a basis of $\operatorname{Gr}_{k}^{F} H^{0}\left(E, \mathcal{O}_{\lambda}(* D)\right)=F_{k} / F_{k-1}$. From this fact and the assumptions $c_{i} \notin \mathbf{Z}(i=1, \ldots, n)$, we can check that the induced homomorphism

$$
\mathrm{Gr}_{k}^{F} \nabla: \operatorname{Gr}_{k}^{F} H^{0}\left(E, \mathcal{O}_{\lambda}(* D)\right) \rightarrow \operatorname{Gr}_{k+1}^{F} H^{0}\left(E, \Omega_{\lambda}^{1}(* D)\right)
$$

is an isomorphism. Hence we have

$$
H^{0}\left(E, \Omega_{\lambda}^{1}(* D)\right) / \nabla H^{0}\left(E, \mathcal{O}_{\lambda}(* D)\right) \cong F_{1} H^{0}\left(E, \Omega_{\lambda}^{1}(* D)\right)=H^{0}\left(E, \Omega_{\lambda}^{1}(D)\right),
$$

which concludes the assertion (i).

Next, we prove (ii). We introduce another filtration to $H^{0}\left(E, \Omega_{\lambda}^{p}(* D)\right)$ :

$$
F_{k}^{\prime} H^{0}\left(E, \Omega_{\lambda}^{p}(* D)\right)=H^{0}\left(E, \Omega_{\lambda}^{p}\left(D^{\prime}+(k-1) D\right)\right), k=0,1,2, \ldots
$$

For $\lambda \in P$, we define $n+1$ functions $\varphi_{j}(u ; \lambda)(j=0, \ldots, n)$ by

$$
\begin{aligned}
\varphi_{0}(u ; \lambda) & =-\lambda \mathfrak{s}\left(u-t_{1} ; \lambda\right), \\
\varphi_{1}(u ; \lambda) & =\frac{\partial \mathfrak{s}}{\partial u}\left(u-t_{1} ; \lambda\right), \\
\varphi_{j}(u ; \lambda) & =\mathfrak{s}\left(u-t_{j} ; \lambda\right)-\mathfrak{s}\left(u-t_{1} ; \lambda\right), j=2, \ldots, n .
\end{aligned}
$$

Here by $\varphi_{j}(u ; 0)$ we understand $\varphi_{j}(u ; 0)=\lim _{\lambda \rightarrow 0} \varphi_{j}(u ; \lambda)$. This definition is valid because $\mathfrak{s}(u ; \lambda)$ is expanded as follows to a Laurent series with respect to $\lambda$ :

$$
\mathfrak{s}(u ; \lambda)=-\frac{1}{\lambda}+\rho(u)+\cdots
$$


where $\rho(u)=\theta^{\prime}(u) / \theta(u)$. Then we see that $F_{0}^{\prime} H^{0}\left(E, \mathcal{O}_{\lambda}(* D)\right)=\mathbf{C} \varphi_{0}(u ; \lambda)$, and that $\left\{\frac{\partial^{k-1}}{\partial u^{k-1}} \varphi_{i}(u ; \lambda)\right\}_{i=1}^{n}$ forms a basis of $\operatorname{Gr}_{k}^{F^{\prime}} H^{0}\left(E, \mathcal{O}_{\lambda}(* D)\right)$. The induced homomorphism

$$
\operatorname{Gr}_{k}^{F^{\prime}} \nabla: \operatorname{Gr}_{k}^{F^{\prime}} H^{0}\left(E, \mathcal{O}_{\lambda}(* D)\right) \rightarrow \operatorname{Gr}_{k+1}^{F^{\prime}} H^{0}\left(E, \Omega_{\lambda}^{1}(* D)\right)
$$

is an isomorphism for $k \geq 1$. Hence we have

$$
H^{0}\left(E, \Omega_{\lambda}^{1}(* D)\right) / \nabla H^{0}\left(E, \mathcal{O}_{\lambda}(* D)\right) \cong F_{1}^{\prime} H^{0}\left(E, \Omega_{\lambda}^{1}(* D)\right) / \nabla F_{0}^{\prime} H^{0}\left(E, \mathcal{O}_{\lambda}(* D)\right) .
$$

Noting that $\nabla \varphi_{0}(u ; \lambda) \neq 0$, we can conclude the assertion (ii).

Remark 2.8. In fact, we have

$$
\begin{aligned}
\nabla \varphi_{0}(u ; \lambda)=( & \left.2 \pi \sqrt{-1} c_{0}-c_{1} \rho(\lambda)+\sum_{j=2}^{n} c_{j} \mathfrak{s}\left(t_{j}-t_{1} ; \lambda\right)\right) \varphi_{0}(u ; \lambda) d u \\
& +\left(c_{1}-1\right) \lambda \varphi_{1}(u ; \lambda) d u-\lambda \sum_{j=2}^{n} \mathfrak{s}\left(t_{j}-t_{1} ; \lambda\right) \varphi_{j}(u ; \lambda) d u .
\end{aligned}
$$

Corollary 2.9. For any $\lambda \in P$, we have $\operatorname{dim} H^{1}\left(M, \mathcal{L} \otimes_{\mathbf{C}} R_{\lambda}\right)=n$. For $\lambda \in P \backslash\{0\}$, the set of $n$ classes $\left\{\left[\mathfrak{s}\left(u-t_{i} ; \lambda\right) d u\right]\right\}_{i=1}^{n}$ forms a basis of $H^{1}\left(M, \mathcal{L} \otimes_{\mathbf{C}} R_{\lambda}\right)$. For $\lambda \in P$, the set of $n+1$ classes $\left\{\left[\varphi_{i}(u ; \lambda) d u\right]\right\}_{i=0}^{n}$ generates the $\mathbf{C}$-vector space $H^{1}\left(M, \mathcal{L} \otimes_{\mathbf{C}} R_{\lambda}\right)$, and these classes are subject to a single relation

$$
\begin{aligned}
& \left(2 \pi \sqrt{-1} c_{0}-c_{1} \rho(\lambda)+\sum_{j=2}^{n} c_{j} \mathfrak{s}\left(t_{j}-t_{1} ; \lambda\right)\right)\left[\varphi_{0}(u ; \lambda) d u\right] \\
& \quad+\left(c_{1}-1\right) \lambda\left[\varphi_{1}(u ; \lambda) d u\right]-\lambda \sum_{j=2}^{n} \mathfrak{s}\left(t_{j}-t_{1} ; \lambda\right)\left[\varphi_{j}(u ; \lambda) d u\right]=0 .
\end{aligned}
$$

In particular, for $\lambda=0$, we have

$$
\begin{aligned}
& \varphi_{0}(u ; 0) d u=d u, \\
& \varphi_{1}(u ; 0) d u=\rho^{\prime}\left(u-t_{1}\right) d u, \\
& \varphi_{j}(u ; 0) d u=\left(\rho\left(u-t_{j}\right)-\rho\left(u-t_{1}\right)\right) d u, \quad j=2, \ldots, n,
\end{aligned}
$$

and the 1-form $\rho^{\prime}\left(u-t_{1}\right) d u$ has a pole at $u=t_{1}$ of order two.

\section{TWisted HOMOLOGY GROUPS}

Let $\check{\mathcal{L}}$ and $\check{R}_{\lambda}$ be the local systems dual to $\mathcal{L}$ and $R_{\lambda}$ respectively. The universal coefficient theorem implies that the natural pairings $H_{i}\left(M, \check{\mathcal{L}}_{\mathbf{C}} \check{R}_{\lambda}\right) \times H^{i}\left(M, \mathcal{L} \otimes_{\mathbf{C}}\right.$ $\left.R_{\lambda}\right) \rightarrow \mathbf{C}(i=0,1,2)$ are non-degenerate. From the results of the last section, we have

$$
\begin{gathered}
H_{0}\left(M, \check{\mathcal{L}} \otimes_{\mathbf{C}} \check{R}_{\lambda}\right)=H_{2}\left(M, \check{\mathcal{L}} \otimes_{\mathbf{C}} \check{R}_{\lambda}\right)=0, \\
\operatorname{dim} H_{1}\left(M, \check{\mathcal{L}} \otimes_{\mathbf{C}} \check{R}_{\lambda}\right)=n .
\end{gathered}
$$

In this section, let us find generators of $H_{1}\left(M, \check{\mathcal{L}} \otimes_{\mathbf{C}} \check{R}_{\lambda}\right)$ and relations among them. Let $\tilde{M}$ be the manifold with circle boundaries which is homotopy equivalent to $M$. We give a cell decomposition of $\tilde{M}$ in Figure 1 is a 2 -cell, $l_{0}, l_{2}, \ldots, l_{n}, l_{\infty}$, $s_{2}, \ldots, s_{n}, m_{0}, m_{1}, m_{2}, m_{3}$ are 1-cells, and the dots •'s stand for 0-cells. Then we 


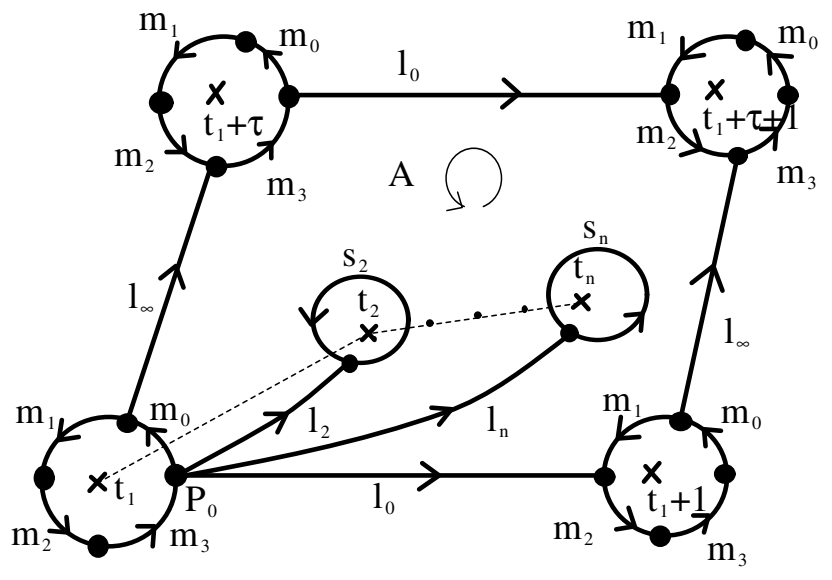

: branch cut for the multi-valuedness of $\mathrm{T}(\mathrm{u})$

Figure 1. Cell decomposition of $\tilde{M}$.

see that the following $n+1$ elements generate the group $Z_{1}\left(M, \check{\mathcal{L}} \otimes_{\mathbf{C}} \check{R}_{\lambda}\right)$ of 1-cycles:

$$
\begin{aligned}
\gamma_{\infty}= & \operatorname{reg}\left(t_{1}, t_{1}+\tau\right)=l_{\infty}+\frac{1-e^{-2 \pi \sqrt{-1} c_{\infty}}}{e^{2 \pi \sqrt{-1} c_{1}}-1}\left(m_{0}+m_{3}\right) \\
& +\frac{1-e^{2 \pi \sqrt{-1}\left(c_{1}-c_{\infty}\right)}}{e^{2 \pi \sqrt{-1} c_{1}}-1}\left(m_{1}+m_{2}\right) \\
\gamma_{0}= & \operatorname{reg}\left(t_{1}, t_{1}+1\right)=l_{0}+\frac{1-e^{-2 \pi \sqrt{-1} c_{0}}}{e^{2 \pi \sqrt{-1} c_{1}}-1}\left(m_{0}+e^{2 \pi \sqrt{-1} c_{1}} m_{1}\right) \\
& +\frac{e^{2 \pi \sqrt{-1} c_{1}}-e^{2 \pi \sqrt{-1} c_{0}}}{e^{2 \pi \sqrt{-1} c_{1}}-1}\left(m_{2}+m_{3}\right) \\
\gamma_{j}= & \operatorname{reg}\left(t_{1}, t_{j}\right)=l_{j}-\frac{s_{j}}{e^{2 \pi \sqrt{-1} c_{j}-1}} \\
& +\frac{m_{0}+e^{2 \pi \sqrt{-1} c_{1}}\left(m_{1}+m_{2}+m_{3}\right)}{e^{2 \pi \sqrt{-1} c_{1}}-1}, j=2, \ldots, n
\end{aligned}
$$

where we put $c_{\infty}=-\lambda-c_{0} \tau-c_{1} t_{1}-\cdots-c_{n} t_{n}$ and the $\operatorname{symbol} \operatorname{reg}(a, b)$ denotes the regularization of the path joining the two points $a$ and $b$. On the other hand, the group $B_{1}\left(M, \check{\mathcal{L}} \otimes_{\mathbf{C}} \check{R}_{\lambda}\right)$ of 1-boundaries is generated by the single element

$\partial A=\left(e^{2 \pi \sqrt{-1} c_{0}}-1\right) \gamma_{\infty}+\left(1-e^{-2 \pi \sqrt{-1} c_{\infty}}\right) \gamma_{0}-\sum_{j=2}^{n} e^{-2 \pi \sqrt{-1}\left(c_{1}+\cdots+c_{j}\right)}\left(1-e^{2 \pi \sqrt{-1} c_{j}}\right) \gamma_{j}$.

Theorem 3.1. Under the assumptions $c_{i} \notin \mathbf{Z}, i=1, \ldots, n$, the twisted homology group $H_{1}\left(M, \check{\mathcal{L}} \otimes_{\mathbf{C}} \check{R}_{\lambda}\right)$ is generated by $n+1$ twisted cycles $\gamma_{\infty}, \gamma_{0}, \gamma_{2}, \ldots, \gamma_{n}$ subject to the unique relation

$\left(e^{2 \pi \sqrt{-1} c_{0}}-1\right) \gamma_{\infty}+\left(1-e^{-2 \pi \sqrt{-1} c_{\infty}}\right) \gamma_{0}-\sum_{j=2}^{n} e^{-2 \pi \sqrt{-1}\left(c_{1}+\cdots+c_{j}\right)}\left(1-e^{2 \pi \sqrt{-1} c_{j}}\right) \gamma_{j}=0$ 


\section{Connection on $H^{1}\left(M, \mathcal{L} \otimes_{\mathbf{C}} R_{\lambda}\right)$}

We put $\mathcal{T}=\mathbf{C}^{n} \times \mathbf{H} \backslash D_{\mathcal{T}}$, where $D_{\mathcal{T}}$ is the divisor on $\mathbf{C}^{n} \times \mathbf{H}$ defined by

$$
D_{\mathcal{T}}=\left\{\left(t_{1}, \ldots, t_{n}, \tau\right) \in \mathbf{C}^{n} \times \mathbf{H} \mid \exists i \neq j \text { s.t. } t_{i}+\Gamma=t_{j}+\Gamma\right\} .
$$

Let $f: \mathcal{M} \rightarrow \mathcal{T}$ be the family of curves over $\mathcal{T}$ whose fiber over $\left(t_{1}, \ldots, t_{n}, \tau\right) \in \mathcal{T}$ is $M\left(t_{1}, \ldots, t_{n}, \tau\right)$. The local system $\mathcal{L} \otimes_{\mathbf{C}} R_{\lambda}$ on $M$ extends naturally to the one on the total space $\mathcal{M}$ under the constraint $\lambda+c_{0} \tau+c_{1} t_{1}+\cdots+c_{n} t_{n}+c_{\infty}=0$ for a constant $c_{\infty} \in \mathbf{C}$, which we denote by the same symbol $\mathcal{L} \otimes_{\mathbf{C}} R_{\lambda}$ by abuse of notation. The coherent $\mathcal{O}_{\mathcal{T}}$-module $R^{1} f_{*}\left(\mathcal{L} \otimes_{\mathbf{C}} R_{\lambda}\right) \otimes_{\mathbf{C}} \mathcal{O}_{\mathcal{T}}$ is locally free, and we have the isomorphism

$$
R^{1} f_{*}\left(\mathcal{L} \otimes_{\mathbf{C}} R_{\lambda}\right) \otimes_{\mathbf{C}} \mathcal{O}_{\mathcal{T}} \cong f_{*} \Omega_{\mathcal{M} / \mathcal{T}, \lambda}^{1}(* D) / \nabla_{\mathcal{M} / \mathcal{T}} f_{*} \mathcal{O}_{\mathcal{M}, \lambda}(* D),
$$

where $\nabla_{\mathcal{M} / \mathcal{T}}=d_{\mathcal{M} / \mathcal{T}}+\omega$. We wish to describe a connection on the right-hand side of (4.1) whose horizontal sections form the image of $R^{1} f_{*}\left(\mathcal{L} \otimes_{\mathbf{C}} R_{\lambda}\right)$, namely the Gauss-Manin connection on the de Rham cohomology [13].

For a local section $\varphi(u ; \lambda) d u$ of the sheaf $f_{*} \Omega_{\mathcal{M} / \mathcal{T}, \lambda}^{1}(* D)$, we define differential operators $\nabla_{t_{i}}(i=1, \ldots, n)$ and $\nabla_{\tau}$ by

$$
\begin{aligned}
\nabla_{t_{i}} \varphi(u ; \lambda) d u= & \frac{\partial \varphi}{\partial t_{i}}(u ; \lambda) d u+\omega_{i}(u) \varphi(u ; \lambda) d u-c_{i} \frac{\partial \varphi}{\partial \lambda}(u ; \lambda) d u \\
\nabla_{\tau} \varphi(u ; \lambda) d u= & \frac{\partial \varphi}{\partial \tau}(u ; \lambda) d u+\omega_{0}(u) \varphi(u ; \lambda) d u-c_{0} \frac{\partial \varphi}{\partial \lambda}(u ; \lambda) d u \\
& +\frac{1}{2 \pi \sqrt{-1}} \frac{\partial}{\partial \lambda} \nabla_{\mathcal{M} / \mathcal{T}} \varphi(u ; \lambda),
\end{aligned}
$$

where we put $\omega_{i}(u)=\left(\partial / \partial t_{i}\right) \log T(u)$ and $\omega_{0}(u)=(\partial / \partial \tau) \log T(u)$.

Proposition 4.1. The differential operators $\nabla_{t_{i}}$ and $\nabla_{\tau}$ define $\mathbf{C}$-endomorphisms on $f_{*} \Omega_{\mathcal{M} / \mathcal{T}, \lambda}^{1}(* D)$ and $\nabla_{\mathcal{M} / \mathcal{T}} f_{*} \mathcal{O}_{\mathcal{M}, \lambda}(* D)$. Consequently, $\nabla_{t_{i}}$ and $\nabla_{\tau}$ induce differential operators on $f_{*} \Omega_{\mathcal{M} / \mathcal{T}, \lambda}^{1}(* D) / \nabla_{\mathcal{M} / \mathcal{T}} f_{*} \mathcal{O}_{\mathcal{M}, \lambda}(* D)$.

Proof. Note that $\varphi(u ; \lambda)$ has the following quasi-periodicity:

$$
\varphi(u+1 ; \lambda)=\varphi(u ; \lambda), \varphi(u+\tau ; \lambda)=e^{2 \pi \sqrt{-1} \lambda} \varphi(u ; \lambda)
$$

from which we see that $\nabla_{t_{i}} \varphi(u ; \lambda) d u$ is also a section of $f_{*} \Omega_{\mathcal{M} / \mathcal{T}, \lambda}^{1}(* D)$. Besides, we have $\nabla_{t_{i}} \nabla_{\mathcal{M} / \mathcal{T}}=\nabla_{\mathcal{M} / \mathcal{T}} \nabla_{t_{i}}$, which implies that $\nabla_{t_{i}}\left(\nabla_{\mathcal{M} / \mathcal{T} f_{*}} \mathcal{O}_{\mathcal{M}, \lambda}(* D)\right) \subset$ $\nabla_{\mathcal{M} / \mathcal{T}} f_{*} \mathcal{O}_{\mathcal{M}, \lambda}(* D)$. We can also prove the assertions for $\nabla_{\tau}$ in a similar way.

For $\lambda \in P \backslash\{0\}$, we take the basis $\left\{\mathfrak{s}\left(u-t_{i} ; \lambda\right) d u\right\}_{i=1}^{n}$ of $H^{1}\left(M, \mathcal{L} \otimes_{\mathbf{C}} R_{\lambda}\right)$ and fix a basis $\left\{\gamma_{(1)}, \ldots, \gamma_{(n)}\right\}$ of $H_{1}\left(M, \check{\mathcal{L}} \otimes_{\mathbf{C}} \check{R}_{\lambda}\right)$. We consider the pairing

$$
f_{i}^{(j)}\left(t_{1}, \ldots, t_{n}, \tau\right)=\left\langle\gamma_{(j)}, \mathfrak{s}\left(u-t_{i} ; \lambda\right) d u\right\rangle .
$$

By the well-known procedure, this pairing is written by the integral

$$
f_{i}^{(j)}\left(t_{1}, \ldots, t_{n}, \tau\right)=\int_{\gamma_{(j)}} T(u) \mathfrak{s}\left(u-t_{i} ; \lambda\right) d u .
$$

Then, by the action of the Gauss-Manin connection, it follows that

$$
\mathbf{f}^{(j)}=\left(\begin{array}{c}
f_{1}^{(j)} \\
\vdots \\
f_{n}^{(j)}
\end{array}\right)
$$


$(j=1, \ldots, n)$ form a fundamental system of solutions to a system of linear partial differential equations with respect to the independent variables $t_{1}, \ldots, t_{n}, \tau$. Such a differential system is given by the following (see [15] for details):

$$
\left\{\begin{array}{l}
\frac{\partial f_{i}}{\partial t_{j}}=-c_{j} \mathfrak{s}\left(t_{j}-t_{i} ; \lambda\right) f_{j}+c_{j} \rho\left(t_{j}-t_{i}\right) f_{i}, \quad j \neq i \\
\frac{\partial f_{i}}{\partial t_{i}}=\sum_{k \neq i} c_{k} \mathfrak{s}\left(t_{k}-t_{i} ; \lambda\right) f_{k}+\left(2 \pi \sqrt{-1} c_{0}-\sum_{k \neq i} c_{k} \rho\left(t_{k}-t_{i}\right)\right) f_{i}, \\
2 \pi \sqrt{-1} \frac{\partial f_{i}}{\partial \tau}=\sum_{k=1}^{n} \frac{c_{k}}{2}\left(\rho\left(t_{i}-t_{k}\right)^{2}-\wp\left(t_{i}-t_{k}\right)\right) f_{i}+\sum_{k=1}^{n} c_{k} \frac{\partial \mathfrak{s}}{\partial \lambda}\left(t_{k}-t_{i} ; \lambda\right) f_{k},
\end{array}\right.
$$

where $\rho(u)=\theta^{\prime}(u) / \theta(u)$, and we assume that $\lambda$ depends on $t_{1}, \ldots, t_{n}, \tau$ such that $\lambda+c_{0} \tau+c_{1} t_{1}+\cdots+c_{n} t_{n}+c_{\infty}=0$ for some constant $c_{\infty}$. This is the system of differential equations satisfied by the Riemann-Wirtinger integral (1.1).

\section{The Riemann-Wirtinger integral and integral Formulae FOR SOLUTIONS OF THE KZB EQUATIONS}

Felder and Varchenko [9] showed that horizontal sections of the KZB connection admit integral representations which are multiple integrals over a direct product of elliptic curves obtained by integrating power products of theta functions. The Riemann-Wirtinger integral is related to the $\mathfrak{s l}_{2}$-case of the KZB equation. We put

$$
T(u)=e^{2 \pi \sqrt{-1} c_{0} u} \theta\left(u-t_{1}\right)^{c_{1}} \cdots \theta\left(u-t_{n}\right)^{c_{n}} ;
$$

here we do not assume $\sum_{j=1}^{n} c_{j}=0$. Take a function $g(\lambda, \tau)$ which satisfies the following partial differential equation:

$$
4 \pi \sqrt{-1} \frac{\partial g}{\partial \tau}(\lambda, \tau)=-\sum_{j=1}^{n} c_{j} \frac{\partial^{2} g}{\partial \lambda^{2}}(\lambda, \tau)+4 \pi \sqrt{-1} c_{0} \frac{\partial g}{\partial \lambda}(\lambda, \tau) .
$$

Then we have

Proposition 5.1. Put

$f_{i}=f_{i}\left(t_{1}, \ldots, t_{n}, \tau ; \lambda\right)=\int_{\gamma} T(u) g\left(\lambda-\sum_{j=1}^{n} c_{j}\left(u-t_{j}\right), \tau\right) \mathfrak{s}\left(u-t_{i} ; \lambda\right) d u, \quad i=1, \ldots, n$.

Then $f_{i}(i=1, \ldots, n)$ satisfy the following system of partial differential equations:

$$
\left\{\begin{array}{c}
\frac{\partial f_{i}}{\partial t_{j}}=c_{j} \frac{\partial f_{i}}{\partial \lambda}-c_{j} \mathfrak{s}\left(t_{j}-t_{i} ; \lambda\right) f_{j}+c_{j} \rho\left(t_{j}-t_{i}\right) f_{i}, \quad j \neq i \\
\frac{\partial f_{i}}{\partial t_{i}}=-\sum_{k \neq i} c_{k} \frac{\partial f_{i}}{\partial \lambda}+\sum_{k \neq i} c_{k} \mathfrak{s}\left(t_{k}-t_{i} ; \lambda\right) f_{k}+\left(2 \pi \sqrt{-1} c_{0}-\sum_{k \neq i} c_{k} \rho\left(t_{k}-t_{i}\right)\right) f_{i} \\
2 \pi \sqrt{-1} \frac{\partial f_{i}}{\partial \tau}=2 \pi \sqrt{-1} c_{0} \frac{\partial f_{i}}{\partial \lambda}-\sum_{k=1}^{n} \frac{c_{k}}{2} \frac{\partial^{2} f_{i}}{\partial \lambda^{2}}+\sum_{k=1}^{n} c_{k} \frac{\partial \mathfrak{s}}{\partial \lambda}\left(t_{k}-t_{i} ; \lambda\right) f_{k} \\
+\sum_{k=1}^{n} \frac{c_{k}}{2}\left(\rho\left(t_{i}-t_{k}\right)^{2}-\wp\left(t_{i}-t_{k}\right)+\frac{1}{3} \frac{\theta^{\prime \prime \prime}}{\theta^{\prime}}\right) f_{i} .
\end{array}\right.
$$


Proposition 5.1 includes a particular case of the results in [9]. We take the Lie algebra $\mathfrak{g}$ in [9] as $\mathfrak{g}=\mathfrak{s l}_{2}$. Let $\alpha$ be the positive simple root of $\mathfrak{s l}_{2}$ with $(\alpha, \alpha)=2$, and let $\Lambda_{1}, \ldots, \Lambda_{n}$ be dominant integral weights with $\left(\Lambda_{1}+\cdots+\Lambda_{n}, \alpha\right)=2$. If we put $c_{0}=0$ and $c_{j}=-\left(\Lambda_{j}, \alpha\right) / \kappa$, then the system of partial differential equations (5.3) essentially coincides with the KZB equation. We can prove Proposition 5.1 by a manner similar to that of Proposition 9 in 9 . Here we remark that we can eliminate the parameter $c_{0}$ from (5.3) if $\sum_{j=1}^{n} c_{j} \neq 0$ because if $g(\lambda, \tau)$ solves the equation (5.1), then $\tilde{g}(\lambda, \tau)=e^{-\pi \sqrt{-1} c_{0}\left(2 \lambda+c_{0} \tau\right) / \sum_{j=1}^{n} c_{j}} g(\lambda, \tau)$ solves the equation (5.1) for $c_{0}=0$ and correspondingly, if $f_{1}, \ldots, f_{n}$ satisfy the system (5.3), then $\tilde{f}_{1}, \ldots, \tilde{f}_{n}$ given by

$$
\tilde{f}\left(t_{1}, \ldots, t_{n}, \tau ; \lambda\right)=e^{-\pi \sqrt{-1} c_{0}\left(2 \lambda+c_{0} \tau+2 \sum_{j=1}^{n} c_{j} t_{j}\right) / \sum_{j=1}^{n} c_{j}} f_{i}\left(t_{1}, \ldots, t_{n}, \tau ; \lambda\right)
$$

satisfy the system (5.3) for $c_{0}=0$.

If we assume $\sum_{j=1}^{n} c_{j}=0$, and $\lambda$ depends on $t_{1}, \ldots, t_{n}, \tau$ such that $\lambda+c_{0} \tau+$ $c_{1} t_{1}+\cdots+c_{n} t_{n}+c_{\infty}=0$ for some constant $c_{\infty}$, the system (5.3) turns into the system (4.2). On the other hand, for $\sum_{j=1}^{n} c_{j}=0$, the equation (5.1) is solved by $g(\lambda, \tau)=h\left(\lambda+c_{0} \tau\right)$ for an arbitrary function $h(\cdot)$ of one variable. Under the further assumption that $\lambda+c_{0} \tau+c_{1} t_{1}+\cdots+c_{n} t_{n}+c_{\infty}=0$, we have

$$
g\left(\lambda-\sum_{j=1}^{n} c_{j}\left(u-t_{j}\right), \tau\right)=h\left(\lambda+c_{0} \tau+\sum_{j=1}^{n} c_{j} t_{j}\right)=h\left(-c_{\infty}\right),
$$

which is just a constant, and the integral (5.2) coincides with the integral (1.1). That is, Proposition 5.1] includes the Riemann-Wirtinger integral as a special case.

\section{ACKNOWLEDGMENTS}

The authors thank Koki Ito for illuminating discussions, and H. Kawamuko, S. Kawai, H. Sakai and N. Suzuki for valuable advice. The authors express their gratitude to the anonymous referee, who carefully read the manuscript and gave many valuable suggestions and comments on the relationship between the RiemannWirtinger integral and the KZB equation in Section 5.

\section{REFERENCES}

1. Aomoto, K., Les équations aux différences linéaires et les intégrales des fonctions multiformes, J. Fac. Sci. Univ. Tokyo, 22 (1975), 271-297. MR0508176 (58:22688c)

2. Aomoto, K., On the structure of integrals of power product of linear functions, Sci. Papers, Coll. Gen. Ed., Univ. Tokyo, 27 (1977), 49-61. MR0590052 (58:28651)

3. Aomoto, K., Une correction et un complement à l'article "Les équations aux différences linéaires et les intégrales des fonctions multiformes", J. Fac. Sci. Univ. Tokyo, 26 (1979), 519-523. MR560011 (82h:32013)

4. Aomoto, K., Configurations and invariant Gauss-Manin connections of integrals I, Tokyo J. Math., 5 (1982), 249-287. MR688126 (85k:32022)

5. Aomoto, K., Configurations and invariant Gauss-Manin connections of integrals II, Tokyo J. Math., 6 (1983), 1-24. MR707836 (85i:32017)

6. Aomoto, K. and Kita, M., Theory of Hypergeometric Functions, Springer Monographs in Mathematics, Springer-Verlag, Tokyo, 2011. MR2799182

7. Chandrasekharan, K., Elliptic functions, Springer, 1985. MR808396 (87e:11058)

8. Deligne, P., Équations différentielles à points singuliers réguliers, Lecture Notes in Math. 163, Springer, 1970. MR0417174 (54:5232)

9. Felder, G. and Varchenko, A., Integral representation of solutions of the elliptic KnizhnikZamolodchikov-Bernard equations, Internat. Math. Res. Notices (1995), no. 5, 221-233. MR1333749 (96g:32040) 
10. Forster, O., Lectures on Riemann surfaces, Graduate Texts in Math. 81, Springer, 1981. MR648106 (83d:30046)

11. Gunning, R. C., Lectures on Riemann Surfaces, Princeton Univ. Press, 1966. MR0207977 $(34: 7789)$

12. Ito, K-K., The elliptic hypergeometric functions associated to the configuration space of points on an elliptic curve I: Twisted cycles, J. Math. Kyoto Univ., 49 (2009), no. 4, 719733. MR2591113 (2011d:14061)

13. Katz, N. and Oda, T., On the differentiation of de Rham cohomology classes with respect to parameters, J. Math. Kyoto Univ., 8 (1968), 199-213. MR0237510 (38:5792)

14. Mano, T., Studies on monodromy preserving deformation of linear differential equations on elliptic curves, J. Math. Phys., 50 (2009) (21 pages). MR2573133 (2011b:34261)

15. Mano, T., The Riemann-Wirtinger integral and monodromy-preserving deformation on elliptic curves, Intern. Math. Res. Notices, 2008 (2008) (19 pages). MR2448089 (2009k:14021)

16. Riemann, B., Gesammelte mathematische Werke. In Nachträge, edited by M. Noether and W. Wirtinger. Leipzig, Germany: Teubner, 1902.

17. Watanabe, H., Transformation relations of matrix functions associated to the hypergeometric function of Gauss under modular transformations, J. Math. Soc. Japan, 59 (2007), 113-126. MR:2302665 (2008h:33009)

18. Watanabe, H., Twisted homology and cohomology groups associated to the Wirtinger integral, J. Math. Soc. Japan, 59 (2007), 1067-1080. MR:2370006 (2009b:14036)

19. Watanabe, H., Linear differential relations satisfied by Wirtinger integrals, Hokkaido Math. J., 38 (2009), 83-95. MR.2501895 (2009m:14066)

20. Watanabe, H., On the general transformation of the Wirtinger integral, preprint.

21. Wirtinger, W., Zur Darstellung der hypergeometrischen Function durch bestimmte Integrale, Akad. Wiss. Wien. S.-B. IIa, 111 (1902), 894-900.

22. Wirtinger, W., Eine neue Verallgemeinerung der hypergeometrischen Integrale, Akad. Wiss. Wien. S.-B. IIa, 112 (1903), 1721-1733.

Department of Mathematical Sciences, Faculty of Science, University of the Ryukyus, Nishihara-cho, Okinawa 903-0213, Japan

E-mail address: tmano@math.u-ryukyu.ac.jp

Kitami Institute of Technology, 165, Koencho, Kitami 090-8507, Hokkaido, Japan

E-mail address: hwatanab@cs.kitami-it.ac.jp 